\title{
Integrity Authentication for SQL Query Evaluation on Outsourced Databases: A Survey
}

\author{
Bo Zhang, Boxiang Dong, Wendy Hui Wang
}

\begin{abstract}
Spurred by the development of cloud computing, there has been considerable recent interest in the Database-as-a-Service (DaaS) paradigm. Users lacking in expertise or computational resources can outsource their data and database management needs to a third-party service provider. Outsourcing, however, raises an important issue of result integrity: how can the client verify with lightweight overhead that the query results returned by the service provider are correct (i.e., the same as the results of query execution locally)? This survey focuses on categorizing and reviewing the progress on the current approaches for result integrity of SQL query evaluation in the DaaS model. The survey also includes some potential future research directions for result integrity verification of the outsourced computations.
\end{abstract}

Index Terms-Database-as-a-Service, SQL query, result integrity verification.

\section{INTRODUCTION}

The amount spent by corporations, non-profit organizations, and government agencies in implementing and supporting data management and analytics is considerable. Globally 3500 enterprises spend, on average, $\$ 664,000$ annually on data management [75]. Due to the fast growth of data volumes, the scale of data management systems is increasingly crossing the petabyte barrier [11], [22], [50|. Unfortunately, the ability of organizations to support effective and efficient data management typically lags behind their ability to collect and store the data. The solution of in-house data analytics software is not satisfactory, as it either may not be able to address users' specific data analysis needs or fail to deliver data management services that are easily deployable. On the other hand, hiring in-house data management professionals is not affordable by small and medium-sized organizations that have limited financial budget.

Explosive development of the Internet and advances in networking technology have fueled a new computing paradigm called Database-as-a-Service (DaaS) [37]. In the DaaS paradigm, the clients who own large volumes of data but lack resources to do data management themselves outsource their data as well as the data analysis to a thirdparty database service provider. The service provider offers adequate hardware, software, and network resources to host the clients' databases, and provides technical supports of data management services, including data access, query processing, and dealing with updates. Several industrial organizations such as Amazon, Google and Microsoft are providing cloud-based database services in various forms. For example, Amazon Web Services (AWS) provides computation capacity and data storages via Amazon Elastic Com-

- B. Zhang and H.W. Wang are with the Department of Computer Science, Stevens Institute of Technology, Hoboken, NI, USA.

Email: bzhang41@stevens.edu, hwang4@stevens.edu

- B. Dong is with the Department of Computer Science, Montclair State University, Montclair, NJ, USA.

Email:dongb@montclair.edu pute Cloud (EC2) [1] and Simple Storage Service (S3) [2]. Google provides Cloud SQL [4], a fully-managed database service for relational PostgreSQL and MySQL databases. Microsoft provides cloud database services [8] on Azure cloud [10]. By using these services, the clients can exploit the benefit of mass storage, accelerated processing capacity, and sophisticated data management and analytics at a low cost.

Outsourcing database management to a computationally powerful service provider enables to achieve sophisticated analysis on large volumes of data in a cost effective way. With this architecture, however, the client no longer has direct control over the outsourced data and computations. Without any security guarantee from the service provider, clients have little faith in the security of the received outsourced services. One of the important security issues of DaaS paradigm is the result integrity of the outsourced computations. In many DaaS paradigms, the client typically has a pay-per-use arrangement with the service provider, where the service provider charges the client proportional to the effort involved in the computations. However, the service providers in practice rarely provide sufficient details of the computation efforts that are charged for service fees ${ }^{1}$ Due to the lack of transparency in the current outsourcing services, the service provider is incentivized to improve its revenue by computing with less resource while charging for more. As an example, project managers from SETI@home [9], a well-known volunteer computing grid, have reportedly [42] uncovered attempts by some users to forge the amount of time they have donated in order to move up on the Web listings of top contributors. Indeed, the director for SETI@home is quoted [42] saying that such cheaters comprise roughly $1 \%$ of their users. Besides cheating on

1. Amazon Elastic Compute Cloud (EC2) |1| charges the users by the number of computing hours and the size of transferred data. It does not provide any detail of the involved computations (e.g., bandwidth, computing cycles, etc.) in the bills. Many online discussions [5], [6] have revealed that there are hidden service fees related to the computation details, e.g., how data is located on the servers. 
computations, there are many other reasons that a service provider may return incorrect query results. For instance, the data or the computations may be corrupted due to malware or security break-ins. Or a malicious insider (e.g., a disgruntled employee) could modify the program and/or the query results. Given the fact that many data analytics applications (e.g., fraud detection and business intelligence) are mission critical, it is important to provide efficient and practical methods to enable the client to verify whether the service provider returned correct results of the outsourced database services.

Intuitively, the general-purpose protocols for verifiable computations [32], [64] can support the verification of any arbitrary query. However, due to the generality, these approaches can incur excessive proof construction overhead at the server side [60]. In the last two decades, a large variety of efficient authentication methods have been proposed for specific types of SQL queries. A brief survey of a subset of these methods was presented in |13|. In this survey article, we will give a comprehensive overview of the existing approaches for result integrity authentication of the DaaS paradigm. By doing the survey, we hope to provide a useful resource for both database and security communities.

The rest of the survey is organized as follows. Section 2 overviews the DaaS framework, the integrity verification goals, and a categorization of the existing authentication methods for SQL query evaluation. Section 3 presents two authenticated data structures that are popularly used in authentication. Section 4 introduces the cryptographic background of a set of authentication approaches. Section 5 discusses the existing authentication techniques that return deterministic integrity guarantees of the result correctness. Section 6 presents the methods that can return probabilistic integrity guarantee. Section 7 discusses the existing authentication methods that verify result freshness. Section 8 presents the works that consider result integrity with additional security features (e.g., access control and privacy). Finally, Section 9 concludes the survey and discusses the possible research directions.

\section{OVERVIEW}

In this section, we overview the preliminaries of the survey. We also provide a categorization of the existing authentication methods for outsourced SQL query evaluation.

\subsection{Database-as-a-Service (DaaS) Paradigm}

The concept of the database-as-a-service (DaaS) paradigm is first defined in 37]. A typical database-as-a-service (DaaS) paradigm consists of three entities: (1) the data owner who possesses a large collection of records $D$. Due to the lack of resources, the data owner outsources $D$ to a service provider; (2) the service provider (SP) who provides storage and data management services for the clients; and (3) the client who sends queries to SP. SP executes the queries on the outsourced data, and returns the query results to the client. The client may have limited computational power (e.g., she may launch the verification on mobile devices). The data owner and the client may be the same entity. In this survey, we mainly focus on relational databases and SQL queries, including range queries, joins, and aggregate queries.

\subsection{Verification Goal}

One of the serious concerns of the DaaS paradigm is that the SP may return incorrect query evaluation results, due to various reasons such as server errors or external compromise [81]. The goal of result integrity authentication is to enable the client to verify that for any query $Q$, if the results $R$ returned by SP is the same as by executing $Q$ on the dataset $D$ locally, i.e., if $R=Q(D)$. There are four different authentication goals: authenticity, soundness, completeness, and freshness.

- Authenticity. The results $R$ must be generated from the outsourced dataset $D$ and not being tampered with.

- Soundness. All the records in $R$ must satisfy $Q$. Intuitively, the soundness of $R$ can be measured by the precision of $R$ :

$$
\operatorname{Precision}(R)=\frac{|R \cap Q(D)|}{|R|} .
$$

The query is sound if precision is 1 .

- Completeness. $R$ must include all records that satisfy $Q$. The completeness of $R$ can be measured by recall of $R$ :

$$
\operatorname{Recall}(R)=\frac{|R \cap Q(D)|}{|Q(D)|} .
$$

The query is complete if recall is 1 .

- Freshness. When there are updates on the outsourced dataset $D$, the query $Q$ must be executed against the latest data records, rather than out-ofdate versions. The returned results $R$ only includes the records from the latest dataset.

\subsection{A Categorization of Integrity Authentication Tech- niques}

In this survey, we categorize the existing result integrity authentication approaches into two types, based on the verification guarantee that these approaches can return:

- The deterministic approaches that verify the query results with $100 \%$ certainty.

- The probabilistic approaches that return a probabilistic verification guarantee of the query results.

\subsubsection{Deterministic Authentication}

Consider the query $Q$ and its results $R$, all deterministic authentication solutions rely on proofs of the query results to return a deterministic integrity guarantee. Intuitively, besides the query results $R$, the SP constructs a short proof of $R$, and sends both the proof and $R$ to the client. The proof sometimes is referred as the verification object (VO). The client utilizes the proof to verify that $R$ satisfies certain requirements (e.g., soundness, completeness, and freshness). At a high level, most of the proof-based authentication methods follow the similar procedure below. Before sending the dataset $D$ to the SP, the data owner computes some auxiliary information $A$ of $D$, which is known as an authentication data structure (ADS). Normally the ADS $A$ is much smaller than $D$. The data owner sends both $D$ and $A$ to the SP, while keeping $A$ locally. She may distribute certain information to 


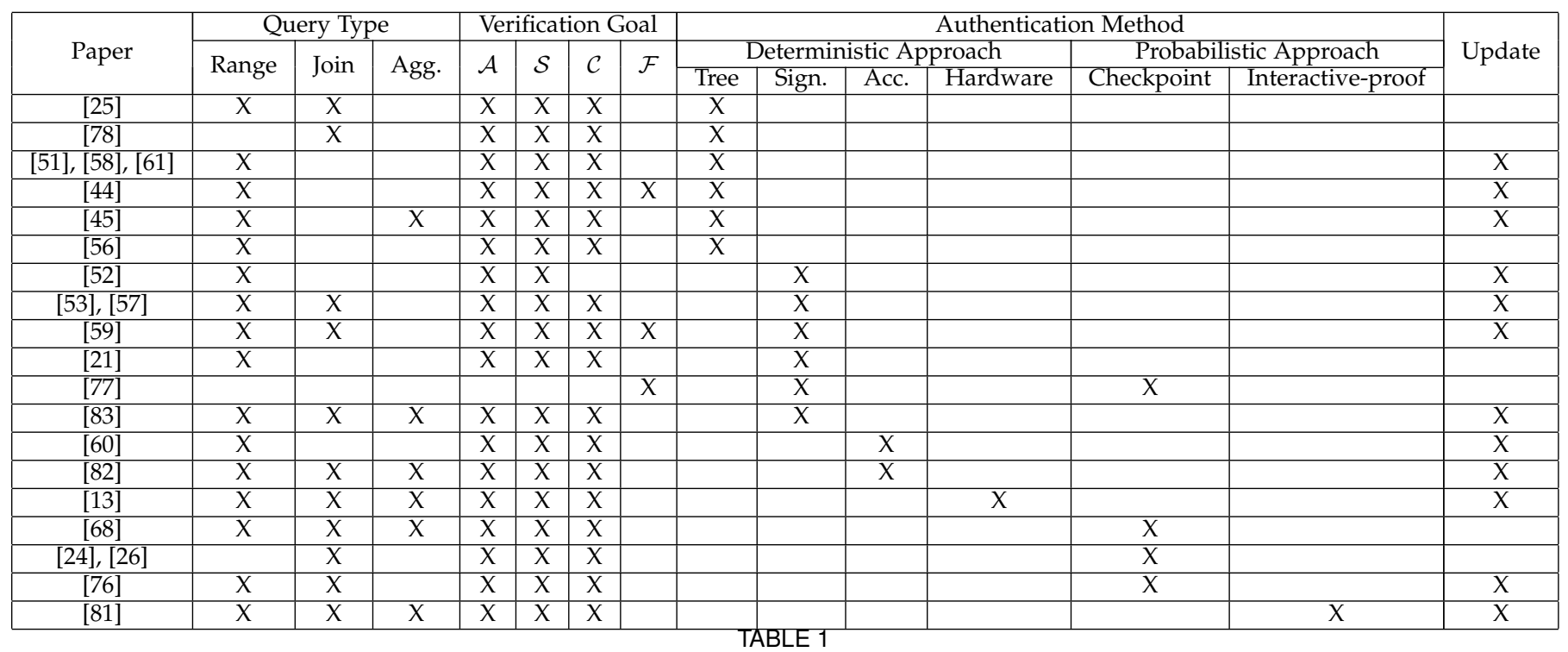

Summary of existing approaches ( $\mathcal{A}$ : authenticity; $\mathcal{S}$ : soundness; $\mathcal{C}$ : completeness; $\mathcal{F}$ : freshness)

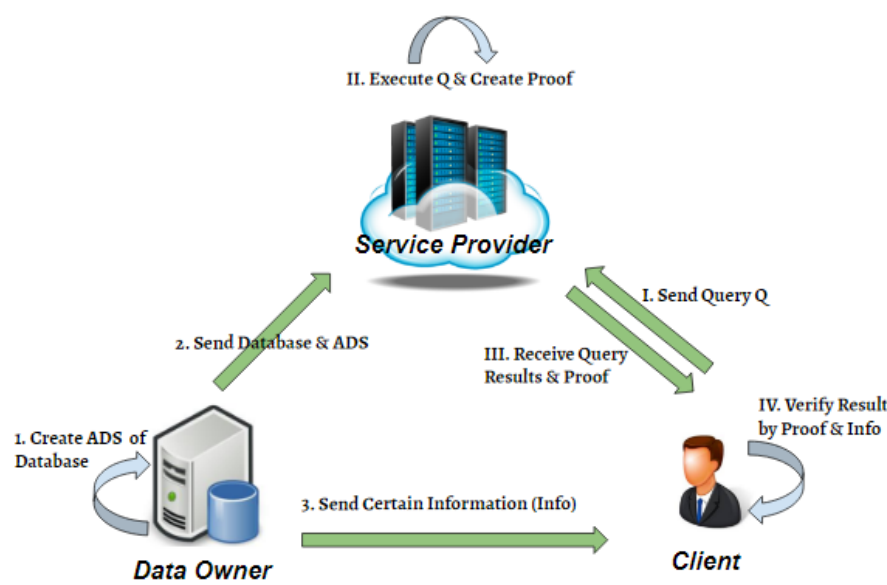

Fig. 1. An example of proof-based authentication method (Steps 1, 2 and 3: Verification preparation phase; Steps I, II, III and IV: Verification phase)

the clients. When a client sends a query $Q$ to the SP, the SP executes $Q$ on $D$, and constructs a proof of the query results $R$, by using both $R$ and the auxiliary information $A$ obtained from the data owner. The SP sends both the query results and the proof to the client. The client verifies result integrity by using both the proof and the certain information that the data owner shared with her. Figure 1 illustrates the framework of the proof-based authentication methods.

Based on how the proof is constructed, we categorize the proof-based methods into four types, namely tree-based solutions, signature-based solutions, accumulation value based solutions, and trusted hardware based solutions.

In the tree-based solutions the ADS normally takes the tree format. As part of query execution, the SP traverses the ADS tree and gathers the information of respective nodes to form the proof. From the query results and the proof, the client re-constructs the traversal path used in query execution and verifies that it is indeed authentic.

By the signature-based methods, the data owner signs individual tuples in a chain fashion before uploading her dataset to the SP. During query execution, the SP gathers the signatures of the tuples in the query results, and compresses these signatures into an aggregated one. The aggregated signature constructed from the authenticated chain forms the proof of the query results. The client can authenticate the results by utilizing the signatures of the tuples in the results and the aggregated signature in the proof.

The accumulation value based solutions are adapted from the cryptographic techniques that authenticate a number of set operations (e.g., intersection, set difference, and set summation). These techniques perform set authentication by constructing a proof of set operation results using accumulation values. The set authentication protocols are then used as building blocks for verification of particular SQL operations (e.g., multi-range selection, join, and aggregation).

The trusted hardware based solutions rely on some tamper-proof, trusted hardware that is deployed on the untrusted server. The original query will be rewritten into sub-queries. All sub-queries that cannot be authenticated are processed inside the trusted hardware, while the results of the remaining sub-queries are verified by other authentication methods, e.g., the tree-based authentication methods.

\subsubsection{Probabilistic Authentication}

The probabilistic authentication methods return a probabilistic result integrity guarantee (i.e., how likely the returned 
results are correct given that they passed the verification). The existing probabilistic authentication methods can be classified into two types: (1) the checkpoint-based solutions, and (2) the interactive-proof based solutions.

The checkpoint-based solutions rely on checkpoints for verification. Unlike the proof-based authentication methods that require the SP to construct the proofs of the query results, the checkpoint-based authentication does not require the involvement of the SP. The key idea of the checkpointbased authentication is to verify if the returned query results include some particular checkpoints (i.e., some specific records). If the results fail the verification based on those checkpoints, the client can determine that the results fail the authentication with $100 \%$ certainty. Otherwise, the client believes that the results pass authentication with some probabilistic guarantee. Most of the checkpoint-based methods construct the checkpoints by inserting counterfeit records $F$ into the dataset $D$ before outsourcing. The assumption is that SP cannot distinguish the real and counterfeit tuples easily. Then for any given query $Q$, a faithful SP should return $Q(F)$ as the part of query results. Thus, any query result that does not include $Q(F)$ can be caught as incorrect.

The interactive proof based solutions extends the information-theoretic interactive proof system [23] to SQL query authentication. The key idea is to translate SQL queries into arithmetic circuits, and verify if the outputs of these circuits are correct. Since the interactive-proof protocol can incur expensive communication and verification overhead, the verification methods takes a random input for the last step of interactive proof protocol, making the authentication method associated with a probabilistic integrity guarantee.

\section{Authenticated Data Structure (ADS)}

In this section, we introduce two prevalent ADS structures, namely, Merkle hash tree (MHT) and Merkle B-tree (MBtree), that are used by a number of existing ADS-based verification methods.

\subsection{Merkle Hash Tree (MHT)}

One of the widely-used ADS structures is Merkle Hash tree (MHT) [65]. A MHT T is a tree in which each leaf node $N$ stores the digest of a tuple $t: h_{N}=H(t)$, where $H()$ is a one-way, collision-resistant hash function (e.g. SHA-1 [31|). For each internal node $N$ of $T$, it is assigned the value $h_{N}=H\left(h_{N_{1}}\|\ldots\| h_{N_{z}}\right)$, where $N_{1}, \ldots, N_{z}$ are the children of $N$, and $\|$ is the concatenation operator. The hash value $h_{\text {root }}$ of the root node is used as the digest of the tree. To serve the authentication purpose, a trusted party (can be the data owner himself) generates the signature of the ADS as $\operatorname{Sig}=\operatorname{Enc}\left(s k, h_{\text {root }}\right)$, where $\operatorname{Enc}()$ is an asymmetric encryption function, and $s k$ is the private key. Sig is shared with the clients for authentication. Figure 2 illustrates an example of MHT.

\subsection{Merkle B-tree (MB-tree)}

Merkle B-tree (MB-tree) [44] enables efficient search on onedimensional data, as it combines MHT with $B^{+}$-tree. In MBtree, the tuples are arranged in the same fashion as standard $B^{+}$-tree. The major difference is the incorporation of hash

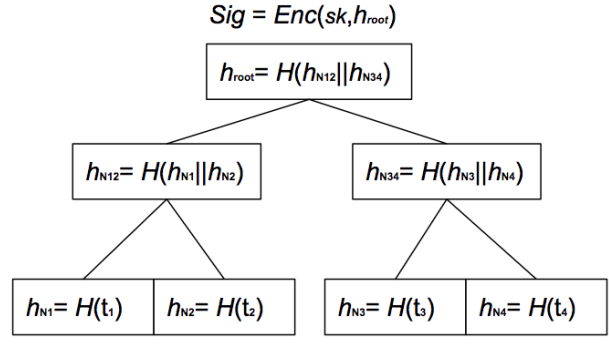

Fig. 2. An example of Merkle-tree

values in the tree nodes. In particular, in the leaf node, each entry is associated with a hash value $h=H(t)$, where $t$ is the tuple in the entry. In the internal node, every pointer to a child node is combined with $h=H\left(h_{1}\|\ldots\| h_{k}\right)$, where $h_{1}, \ldots h_{k}$ are the hash values in the child node.

\section{Cryptographic Background}

In this section, we present the cryptographic background of the signature-based and accumulation value based verification methods.

\subsection{Bilinear Pairing}

Bilinear pairing [49] uses a pairing between two cryptographic groups to a third group with a mapping. In particular, let $G$ and $G_{T}$ be two groups of order $p$ (with $p$ a $\lambda$-bit prime), a bilinear pairing on $\left(G, G_{T}\right)$ is a map $e: G \times G \rightarrow G_{T}$ that satisfies the following conditions:

- Bilinearity: $\forall a, b \in \mathbb{Z}_{1}, P, Q \in G, e\left(P^{a}, Q^{b}\right)=$ $e(P, Q)^{a b}$;

- Non-degeneracy: $e(g, g) \neq 1$, where $g \in G$ is a generator of group $G$;

- Computability: There exists an efficient algorithm to compute $e$.

\subsection{Aggregate Signature}

An aggregate signature scheme is a digital signature that supports aggregation. Informally, given $n$ signatures on $n$ distinct messages, all these signatures are aggregated into a single short signature [18].

\subsubsection{BGLS Scheme}

The BGLS [18| aggregate signature scheme (named after its four authors) relies on bilinear pairing for signature aggregation. In particular, given a bilinear map e $: G \times G \rightarrow G_{T}$ over two groups of prime order $p$, let $s \in \mathbb{Z}$, be a secret key, the public key $v$ is generated as

$$
v=g^{s} \in G,
$$


where $g$ is the generator of group $G$. For any message $m_{i}$, let $H:\{0,1\}^{*} \rightarrow G$ be a full-domain hash function. The signature $\sigma_{i}$ of $m_{i}$ is generated as

$$
\sigma_{i}=H\left(m_{i}\right)^{s}
$$

Clearly, $\sigma_{i} \in G$.

Given $n$ signatures $\left\{\sigma_{1}, \ldots, \sigma_{n}\right\}$ where $\sigma_{i}=H\left(m_{i}\right)^{s_{i}}$, their aggregate signature is generated as

$$
\sigma_{1, n}=\Pi_{i=1}^{n} \sigma_{i}
$$

Again, $\sigma_{1, n}$ is a member in group $G$.

Upon receiving an aggregate signature $\sigma_{1, n}$, the set of messages $\left\{m_{i}\right\}$, and the public keys $\left\{v_{i}\right\}$, whether $\sigma_{1, n}$ corresponds to the messages $\left\{m_{i}\right\}$ can be verified by first computing $h_{i}=H\left(m_{i}\right)$ for $1 \leq i \leq n$. The messages are accepted if

$$
e\left(\sigma_{1, n}, g\right)=\Pi_{i=1}^{n} e\left(h_{i}, v_{i}\right)
$$

\subsubsection{Homomorphic Linear Authentication (HLA) Scheme}

Homomorphic Linear Authentication (HLA) scheme [12 allows to check the authenticity of the messages against the generated aggregate signature without the knowledge of individual messages. The HLA scheme consists of the following algorithms:

- $\quad(s k, p k) \leftarrow \operatorname{KeyGen}\left(1^{\ell}\right)$ : given a security parameter $\ell$, this algorithm outputs a pair of public and private keys $(s k, p k)$.

- $\quad \sigma_{i} \leftarrow \operatorname{Tag} \operatorname{Ten}\left(s k, m_{i}\right)$ : for message $m_{i}$, this algorithm generates a signature $\sigma_{i}$ based on the private key $s k$.

- $\sigma_{1, n} \leftarrow H L A A g g(\vec{c}, T \vec{a} g)$ : this linear aggregation algorithm takes the input of a vector of coefficients $\vec{c}=$ $\left\{c_{1}, \ldots, c_{n}\right\}$ and a vector of tags $\overrightarrow{T a} g=\left\{\sigma_{1}, \ldots, \sigma_{n}\right\}$ with respect to a vector of messages $\vec{m}=\left\{m_{1}\right.$, $\left.\ldots, m_{n}\right\}$. This algorithm produces the aggregate signature $\sigma_{1, n}=\sum_{i=1}^{n} c_{i} m_{i}$.

- $\{0,1\} \leftarrow \operatorname{Vrfy}\left(p k, m^{\prime}, \sigma_{1, n}\right)$ : given the input as the public key $p k$, a candidate message $m^{\prime}$ and an aggregate signature $\sigma_{1, n}$, this algorithm returns 1 if $\sigma_{1, n}$ is valid with respect to $m^{\prime}$, and 0 otherwise.

\subsection{Accumulation Value}

An accumulator scheme [14], [15] allows aggregation of a large set of inputs into one constant-size value. Papamanthou et al. |63| proposed an accumulation value scheme for set operation verification. In particular, given a set of elements $S=\left\{x_{1}, \ldots, x_{n}\right\}$, the accumulation value of $S$ is constructed as:

$$
\operatorname{acc}(S)=g^{\prod_{i=1}^{n}\left(x_{i}+s\right)},
$$

where $g$ is the generator of a bilinear group, and $s$ is a randomly chosen secret value.

For any pair of subsets $S_{1}$ and $S_{2}$ of $S$ such that: (1) $S_{1} \cup S_{2}=S$; and (2) $\left|S_{1}\right|+\left|S_{2}\right|=|S|$, it must be true that $e\left(\operatorname{acc}\left(S_{1}\right), \operatorname{acc}\left(S_{2}\right)\right)=e(\operatorname{acc}(S), g)$, where $e$ is the bilinear pairing function. Based on the discrete log assumption [69], it is NP-hard for any polynomial-time adversary without the knowledge of the secret key $s$ to find a different set $S_{3} \neq S_{2}$ such that $e\left(\operatorname{acc}\left(S_{1}\right), \operatorname{acc}\left(S_{3}\right)\right)=e(\operatorname{acc}(S), g)$.

\subsection{Verification Protocols for Set Operations}

\subsubsection{Set Intersection}

Given a collection of sets $\mathcal{S}=\left\{S_{1}, \ldots, S_{t}\right\}, I=S_{1} \cap S_{2} \cap$ $\cdots \cap S_{t}$ is the correct intersection of $\mathcal{S}$ if and only if:

- $I \subseteq S_{1} \wedge \cdots \wedge \subseteq S_{t}$ (subset condition);

- $\quad\left(S_{1}-I\right) \cap \cdots \cap\left(S_{t}-I\right)=\emptyset$ (completeness condition).

Papamanthou et al. proposed a set intersection verification protocol to verify that $I$ is a correct intersection of $\mathcal{S}$ [63. For each set $S_{i}$, the SP demonstrates $I \subseteq S_{i}$ by providing $\operatorname{acc}\left(W_{i}\right)$, where $W_{i}=S_{i}-I$, and $\operatorname{acc}()$ is the function that calculates the accumulation value (Equation 5). If $e\left(\operatorname{acc}(I), \operatorname{acc}\left(W_{i}\right)\right)=e\left(\operatorname{acc}\left(S_{i}\right), g\right)$ for every set $S_{i}$, where $e$ is the bilinear pairing, the client is assured that the subset condition is met. The intersection completeness is proved by finding a set of polynomials of $s$, namely $\left\{q_{1}, \ldots, q_{t}\right\}$, such that $\sum_{i=1}^{t} P\left(W_{i}\right) q_{i}=1$, where $P\left(W_{i}\right)=\prod_{x_{j} \in W_{i}}\left(x_{j}+s\right)$.

\subsubsection{Set Difference}

Papadopoulos et al. |60| designed a method for efficient authentication of set difference results based on the accumulation values. In particular, given two sets $X_{1}$ and $X_{2}$ such that $X_{2} \subseteq X_{1}$, to demonstrate $X=X_{1} \backslash X_{2}$, the prover constructs $\operatorname{acc}(X)$ (Equation 5). The verifier simply checks $e\left(\operatorname{acc}\left(X_{2}\right), \operatorname{acc}(X) \stackrel{?}{=} e\left(\operatorname{acc}\left(X_{1}\right), g\right)\right.$ for verification, where $e$ is the bilinear pairing, and $\operatorname{acc}()$ is the function that calculates the accumulation value (Equation 5).

\subsubsection{Summation over Sets}

Given a set $S=\left\{x_{1}, \ldots, x_{n}\right\}$, Zhang et al. [82] proposed the sum verification protocol based on accumulation values to check if sum $\stackrel{?}{=} \sum_{i=1}^{n} x_{i}$. Let $M(S)=\prod_{i=1}^{n}\left(x_{i}^{-1}+s\right)=$ $a_{n} s^{n}+\cdots+a_{1} s+a_{0}$. Obviously, $\sum_{i=1}^{n} x_{i}=a_{1} / a_{0}$. Based on this reasoning, the prover provides $a_{0}, a_{1}$, as well as proof that they are the coefficients of the smallest exponent in $M(S)$, for the purpose of verification.

\section{Deterministic Authentication Ap- PROACHES}

In this section, we overview the authentication approaches that use verification object (VO) for authentication. There are three different methods to construct VO: (1) use the authenticated data structure; (2) use signature aggregation and chaining; and (3) use cryptographic accumulation values. All these methods return a deterministic integrity guarantee (i.e., with $100 \%$ certainty). Besides these three methods, we also discuss the verification method that relies on the trusted hardware to return deterministic guarantee. Next, we discuss each of these four different methods respectively.

\subsection{Tree-based Authentication}

Quite a few existing authentication approaches use the authenticated data structure (ADS) to construct the verification objects. Most of these works use different variants of Merkle hash tree (MHT) for specific query types.

Devanbu et al. |25] is one of the first works that use MHT to verify inclusiveness (i.e., soundness) and completeness of SQL query evaluation. They use Merkle hash tree (MHT) to construct the ADS. During query execution, the 


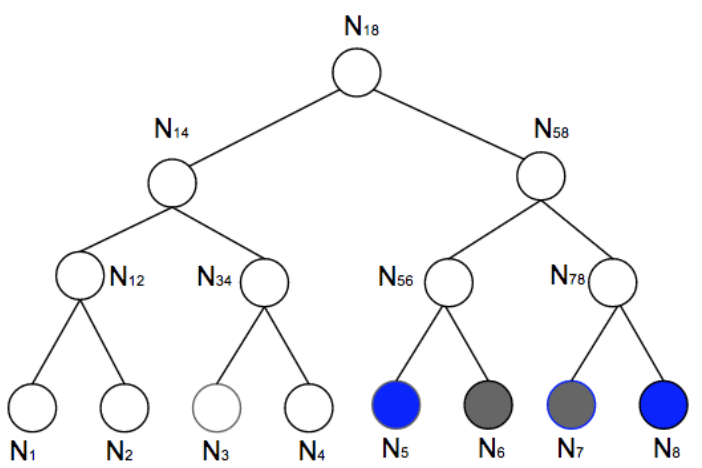

Fig. 3. Illustration of MHT-based Verification 25.

SP traverses the MHT and gathers the respective nodes in the MHT to construct the VO. For instance, given a singledimensional range query $Q$, the $\mathrm{VO}$ includes the lowest common ancestor in MHT that cover the tuples in $Q$ and their nearest lower/upper bound nodes, as well as the nodes in the proximity subtrees that contain the boundary nodes occurring consecutively to the smallest (largest) element of $Q$. Consider the Merkle tree in Figure 3 as an example. Assume the leaf nodes (colored gray) $N_{6}$ and $N_{7}$ falls in the query range. The nearest lower (upper, resp.) bound node is $N_{5}\left(N_{8}\right.$, resp). For VO construction, the SP identifies: (1) node $N_{58}$, which is the lowest common ancestor of nodes $N_{6}$ and $N_{7}$; and (2) the proximity trees that include $N_{58}$ and the two paths from $N_{58}$ to $N_{5}$ and to $N_{8}$. The SP includes the hash value of $N_{58}$ and the proximity trees in VO. The authenticity of $Q$ can be verified by re-constructing the root hash of $N_{58}$ using the hash values of $N_{5}-N_{8}$, which are included in VO. The completeness of $Q$ can be verified via the proximity sub-trees in VO. Devanbu et al. [25] also considered other types of SQL queries, including projection, join, set union and intersection. The key idea is similar to the verification of selection queries. We omit the details for these types of queries. Given a dataset $D$ that contains $n$ tuples and a query $Q$ whose result contains $t$ data points, the VO size of $Q$ by [25] is $O\left(t+\log _{2}(n)\right)$.

One weakness of [25] is that the VO size depends on the size of the query answers and the outsourced dataset. To eliminate this dependence on data and query size, Pang et al. [58] designed a new ADS named verifiable B-tree (VBtree). VB-tree is similar to MB-tree (Section 3.2. It constructs a Merkle hash tree on top of the $B+$-tree by adding the digests on every B-tree node. The digests are computed using a cumulative and commutative hash function. For the VO construction, the SP identifies the smallest subtree (called enveloping tree) in VB-tree that covers all the tuples in the query results. For a given range selection query, the
VO of the selection results includes: (1) the signed digest for the node at the root of the smallest subtree (called enveloping tree) in VB-tree that covers all the result tuples of the query; and (2) the signed digest for each node in the enveloping subtree that represents those branches that do not overlap the result. The result verification is similar to [25]; the client tries to re-construct the signed digest of the root of the VBtree, and matches it with the local copy that is shared by the data owner. Unlike [25] whose VO contains the digests all the way to the root of the tree index, the $\mathrm{VO}$ of [58] only needs to contain proofs for the smallest subtree that envelops the query result. Therefore, the VO size grows linearly with the size of the query results, but is independent of the data size. However, VB-tree cannot be used for the verification of result completeness.

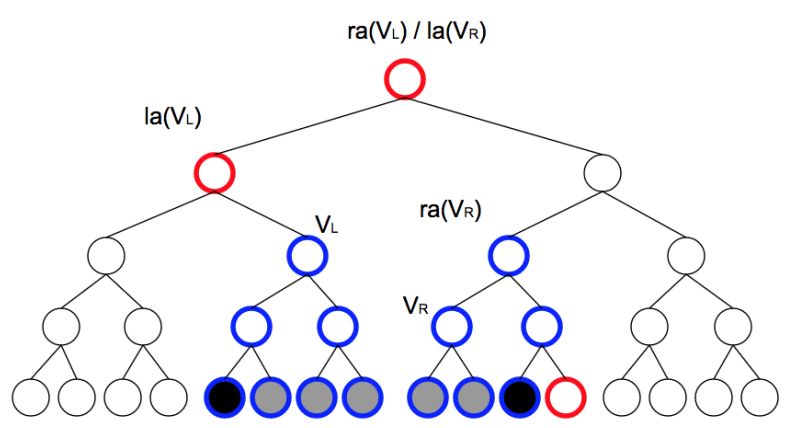

Fig. 4. An example of HAT $(l a(v)$ (resp. $r a(v))$ denotes the least command ancestor of left (resp. right) adjacent nodes of $v$ )

Although VB-tree [58] eliminates the complexity dependency on data size, it may suffer from the strong assumption that the SP must be trusted to some degree [56]. Furthermore, VB-tree uses a one-way function based on modular exponentiation, which was shown infeasible to handle large answer sizes [56]. Therefore, [56] proposed a new ADS named Hybrid Authentication Tree (HAT), which incorporates fast hash functions with an efficient RSA based one-way quasi-commutative accumulator [15]. A HAT is a binary search tree with data stored at the leaves, along with a digest procedure that incorporates a fast collision intractable hash function and an accumulator. In HAT, the hash values of the leaf nodes are generated by applying the standard hash function on the data values, while the hash values of the internal nodes incorporate the hash values from the lowest common ancestors of all left and right adjacent nodes respectively. The final digest value of the HAT tree is com- 
puted as the accumulator of the hash of all the nodes along with the root hash. Given a range query $[a, b], \mathrm{SP}$ returns all data items in the range and two boundary leaves in HAT. The verification is based on the concept of covering nodes, which is a set of nodes with disjoint HAT subtrees whose leaves are the exact answer to the range query. The verification method utilizes the fact that if the two covering nodes $v_{R}$ and $v_{L}$ are adjacent, then their leaf nodes are continuous and no values are missing between the two boundary values of the range $[a, b]$. This is verified by computing the hash values of internal nodes as well as the accumulator proof of the covering nodes and their immediate adjacent left and right nodes returned by SP. Finally, the client checks if the digest of root of the HAT matches the local copy shared by the data owner. Take Figure 4 as an example. The leaf nodes in gray color denote the query results. $V_{L}$ and $V_{R}$ denote the two covering nodes. The SP generates the regular hash value by using the hash function $f_{1}()$ and the accumulate hash value by using the hash function $f_{2}()$. Given a query $Q$, besides the query results, the SP returns the VO that consists of the following information: (1) the nearest lower/upper bound nodes (denoted in black color); (2) the two accumulation values $f_{2}$ (root) excludes the digest of $f_{2}\left(V_{L}\right)$ and $f_{2}\left(V_{R}\right)$ respectively, (3) the digest values that are needed to reconstruct $f_{1}\left(V_{L}\right)$ and $f_{1}\left(V_{R}\right)$; and (4) the digest values that are needed to reconstruct $f_{2}\left(V_{L}\right)$ and $f_{2}\left(V_{R}\right)$. The digest values of the nodes in red (blue, resp.) color circle outline are returned (computed, resp.) by the SP (the client, resp.). With those digest values, the client first verifies that $V_{L}$ and $V_{R}$ are adjacent by checking if the digest of $r a\left(V_{L}\right)$ equals to the digest of $l a\left(V_{R}\right)$. Then it checks if the two accumulation values together with $f_{1}\left(V_{L}\right)$ and $f_{1}\left(V_{R}\right)$ can reconstruct $f_{2}$ (root).

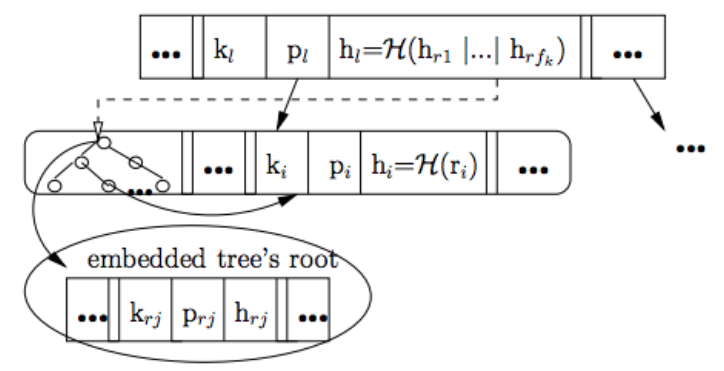

Fig. 5. An example of EMB-Tree Node [4]

So far the existing works mainly consider static scenarios, where owners never issue data updates. Li et al. [44] are one of the first work that designs dynamic ADS for query authentication. They design three ADS structures, namely, the Aggregated Signatures with B+-trees (ASB-tree), the Merkle B-tree (MB-tree), and the embedded MB-tree (EMB-tree). The ASB-tree is a B+-tree whose leaf nodes contain hash values that are constructed from the consecutive pairs of tuples. For example, for the leaf node whose entry corresponds to the tuple $r_{i}$, assume the consecutive tuple of $r_{i}$ is $r_{j}$, based on some sorted order on a given attribute. Then $r_{i}$ corresponds to a leaf node in the ASBtree, which is associated with a pair $\left(r_{i}, s_{i}\right)$, where $s_{i}=$ $S\left(r_{i} \mid r_{j}\right)$ (| denotes some canonical pairing of strings that can be uniquely parsed back into its two components). Chaining tuples in this way enables to verify that there are no tuples missing in-between the lower/upper bound node. The MB-tree has been discussed in Section 3.2 An EMB-tree consists of regular $B^{+}$-tree entries augmented with an embedded MB-tree. Each EMB-tree node consists of a triplet $\left(k_{i}, p_{i}, h_{i}\right)$, where $k_{i}$ is key of the node, $p_{i}$ is the pointer to the embedded Merkle tree at this node, and $h_{i}$ is the hash value of the embedded tree. An example of EMB-trees is shown in Figure 5 . When there are updates on the data, only the path from the affected leaf (i.e., the updated data tuple) to the root is updated. The VO construction and query authentication procedure based on the EMB-tree is similar to [25]. Besides the design of the EMBtree structure, [44] conducted the analysis of some existing approaches, including the aggregated signatures for $\mathrm{B}^{+}$. trees, the Merkle B-tree, and the EMB-trees, over all six metrics: (1) the computation overhead for the owner, (2) the owner-SP communication cost, (3) the storage overhead for the SP, (4) the computation overhead for the SP, (5) the client-SP communication cost, and (6) the computation cost for the client. The experimental results show that, the ASBtree has the highest construction cost, the most expensive $\mathrm{VO}$ construction cost and verification cost. But it has the smallest VO size. The Merkle B-tree has the largest VO size, but the cheapest verification cost and update cost. The EMBtree achieves a good compromise between ADS construction overhead and verification cost.

Most of the existing works discussed so far mainly focus on range queries. Yang et al. [78] initiated the investigation of result authentication of join operations. They proposed three authenticated join algorithms depending on the ADS availability: (1) Authenticated Indexed Sort Merge Join (AISM), which utilizes a single ADS in one of the base relations, (2) Authenticated Index Merge Join (AIM) that requires an ADS for both relations, and (3) Authenticated Sort Merge Join (ASM), which does not rely on any ADS. Consider the join of two tables $R$ and $S$ on the attribute(s) $J$. The AISM algorithm relies on the MB-tree and the rankedlist for authentication of join results of $R$ and $S$. In the pre-processing step, the outer table $R$ is sorted on the join attribute $J$, with the output as a rank list. The rank list outputs the verifiable order by which the client follows to verify the signature of records in the results. Given a set of records $R^{\prime} \subseteq R$, the query of finding the join records of $R^{\prime}$ in $S$ is equivalent to a single range query $Q=\sigma_{c_{l} J c_{u}} S$, where $c_{l}$ and $c_{u}$ are the lower- and upper- bound value of $R^{\prime}$ on the join attribute $J$. The result of the range query evaluation (and thus the join results) can be authenticated by utilizing the MB-tree [44] of $S$ as the ADS. The query authentication 
method is similar to [25]. In order to save communication cost, the AIM algorithm constructs two MB-trees for $R$ and $S$ respectively. Based on the two MB-trees $T_{R}$ and $T_{S}$, the SP alternatively searches the matching tuples on each ADS and prepares the VO. ASM method does not require any ADS at all. It sorts the data values of the join attributes $J$ of $R$ and $S$ as two rank list respectively. Then the two sorted tables $R^{\prime}$ and $S^{\prime}$ are merged as a single rank list, with the records that can be joined marked. Then it creates a bitmap $B_{R}$, in which the bit of the records of $R$ that have join partners in $S$ is set as 1 , otherwise 0 . Both signatures of $R$ and $|R|$, and $B_{R}$ are added to VO. The same process repeats for $S$. The client verifies the result by checking the bitmap values of the received results with VO. Compared with AISM and AIM, ASM is less efficient as it does not utilize any ADS. However, this also brings flexibility for authentication of complex queries such as multi-way joins.

$\mathrm{Li}$ et al. [45] extend the study to aggregation queries. They consider both static and dynamic cases for query authentication. For the static case, first, they show that authenticating SUM queries is equivalent to authenticating prefix sums [38]. To authenticate prefix sum, Li et al. designed a new $\mathrm{A} \overline{\mathrm{DS}}$ structure named the authenticated prefix sums tree (APS-tree), which takes the format of of a multi-way Merkle hash tree, in which each leaf entry is converted into a base$\mathrm{f}$ number. To construct the $\mathrm{VO}$ of a given query $Q$, the $\mathrm{SP}$ returns the $2^{d}$ corner prefix sum values as the part of $\mathrm{VO}$, where $d$ is the number of dimensions of the dataset. Besides, the VO contains the hash values of the APS-tree nodes that are needed to authenticate the sum results, as well as the encoding of the path for each node. Based on the VO, the client can authenticate the query result by authenticating each of the $2^{d}$ elements of the answer set by computing the hash of the root for each path and then comparing it with the local signature generated by the data owner.

The APS-tree only can be used for static databases. It cannot work well for dynamic settings as it may lead to high querying cost. Therefore, Li et al. [45] designed another ADS named authenticated aggregation B-tree (AAB-tree) for the dynamic case. The AAB-tree is an MB-tree with each node associated with an aggregate value as the sum of the aggregate values of its children, and a hash value over the concatenation of both the hash values and the aggregate values of the children. An example of AAB-tree node shows in Figure 6 The AAB-tree can be used for authentication of one-dimensional aggregate queries in a dynamic setting since the owner can easily issue deletions, insertions and updates to the tree, which handles them similarly to a normal B+-tree. It can be easily extended to deal with the multi-dimension aggregate queries in the similar way as the APS-tree. Other than COUNT and AVG, AAB-tree supports authentication of MIN and MAX as well, by replacing the SUM aggregate in each entry with the MIN/MAX aggregate.

Most of the existing ADS-based verification methods involve the data owner into the verification setup for ADS construction, which may not be feasible for the data owner who may have limited computational resources. Furthermore, the VO is typically large, which may bring significant communication overhead. To address these two drawbacks, Stavros Papadopoulos et al. [61] separate authentication

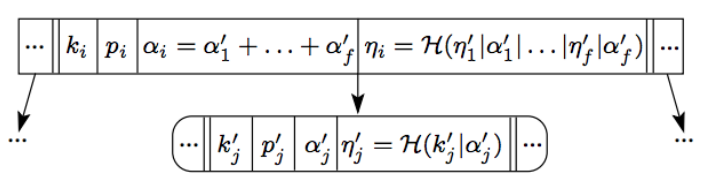

Fig. 6. An example of AAB-tree node [45] ( $k_{i}$ : value range; $p_{i}$ : pointer to the children node; $\alpha_{i}$ : aggregation of all children nodes; $\eta_{i}$ : the node digest.

from query execution by exploiting a trusted entity (TE). The high-level idea is that the data owner sends her dataset to TE. TE generates the digest of each record by using a one-way, collusion-resistant hash function. When the client receives the result of a range query from the $\mathrm{SP}$, the client sends the query to TE. TE evaluates the query on the dataset received from the data owner, and produces a verification token, which is constructed by applying the exclusive-OR (XOR) operator on the digests of tuples in the query result. TE transmits the verification token to the client. The client computes the XOR of the digests of the records in the returned results by the SP, and matches it against the verification token by TE. To facilitate the authentication process, [61] includes the design of a new ADS named XOR $\mathrm{B}$-Tree (X $\mathrm{B}$-Tree) that integrates the B-tree with $\mathrm{XOR}$ values. The $\mathrm{XB}$-tree is very similar to the B-tree; the difference is that each entry of the XB-tree is associated with a bit string that represents the results of XOR operator. An example of XB-tree is shown in Figure 7. Search on the XB-tree and VO construction are very similar to those on the MB-tree.

So far most of the techniques incorporate the Merkle hash tree with a data index to construct ADS. As part of query execution, the SP traverses the ADS and gathers the respective nodes to form the VO. Mouratidis et al. [51] designed a novel scheme called Partially Materialized Digest scheme (PMD) that separates MHT from the data index. Instead, it uses a main index (MI) for querying, and a separate digest index (DI) for query authentication. The MI is a standard $B^{+}$-tree on key. Range queries are evaluated on MI in the same way as on the $B^{+}$-tree. The DI is constructed as follows. First, for each leaf MI node $E_{i}$, a lower MHT is built on top of it. Then the upper MHT is built over the roots of the lower trees. The root of the upper tree is signed with the owner's private key. Similar to [25], for a 


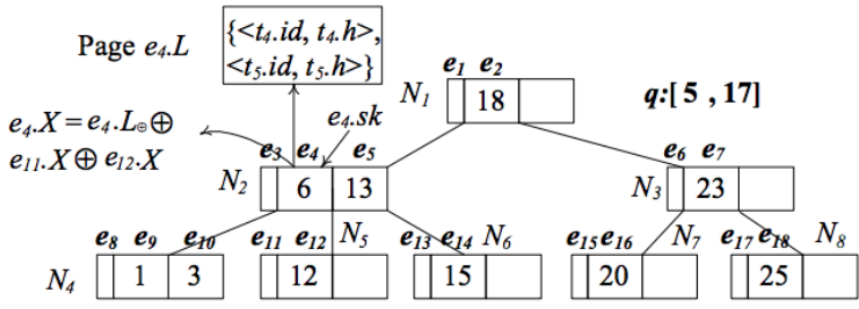

authentication. By their approach, the data owner constructs a standard RSA signature [66] for each record, and sends these signatures together with the dataset. The SP uses the Condensed RSA to compress the RSA signatures |66 that are associated with all records in the query results into a single signature. The single signature then is sent to the client together with the query results as its VO. The client can verify the correctness and authenticity of the returned query results by reconstructing the single signature from the signatures of returned result tuples. The major advantage of this method is that the $\mathrm{VO}$ size is significantly reduced by combining multiple tuple signatures into a single signed message.

Although effective, [52] cannot verify the completeness of the query results. Narasimha et al. [53] extended [52] by combining signature aggregation with signature chaining for the authentication of range queries. Unlike [52 that aggregates the signatures of individual tuples, [53] constructs the $\mathrm{VO}$ from the signature of each individual tuple along with its immediate predecessor, i.e., $\operatorname{sign}\left(H\left(t_{i}|| t_{i-1}\right)\right.$, where $t_{i-1}$ is the immediate predecessor of $t_{i}$ on the search attribute. By including the predecessor in the signature, it forms a chain of tuples ordered on the search attribute. By using the VO, the client verifies that the set of tuples received in the result indeed form a valid chain.

Simultaneously, Pang et al. [57] devised a new signaturebased scheme for range and join queries. It constructs the $\mathrm{VO}$ from the signature of each individual tuple along with both of immediate predecessor and successor, i.e., $\operatorname{sign}\left(H\left(g\left(t_{i-1}\right)\left\|g\left(t_{i}\right)\right\| g\left(t_{i+1}\right)\right)\right)$, where $t_{i-1}$ and $t_{i+1}$ are the immediate predecessor and successor of $t_{i}$ on the search attribute respectively, sign is the digital signature signing function (e.g., RSA or DSA [66]), $H$ is the hash function, $\|$ is the concatenation operator, and $g\left(t_{i}\right)$ is the digest of $t_{i}$. The proposed scheme can support joins on primary key and foreign key, by using signatures on the key attribute of a relation to generate proof of the completeness of query results from that relation.

One weakness of the signature aggregation approach [57] is that it only supports single-dimension range queries on the key attribute, since it requires the tuples to be ordered based on their key attribute values. To address the limitations of the signature chaining approaches in [57], Cheng et al. [21] combined signature aggregation with a data partitioning structure (e.g., R-tree [36] and KD-tree [16]) to support authentication of multi-dimensional range query evaluation. Before outsourcing, the data owner constructs an R-tree or KD-tree of the dataset to get data partitions. Each partition $P$ will be associated with a signature, which is aggregated from the signatures of two auxiliary bounding tuples of $P$. By including the signature of those partitions that do not overlap with the query range, instead of the records in these partitions, it reduces the VO size significantly.

Zheng et al. |83| designed the signature-based authentication for various types of queries, including aggregation queries. It utilizes the Homomorphic Linear Authentication (HLA) (Section 4.2.2) to construct the aggregate signature for authentication of SUM queries. To support authentication of other types of queries including range selection queries, the authentication method combines HLA aggregation sig- 
nature with MB-tree [44], by associating the MB-tree entries with the HLA aggregation signatures.

\subsection{Accumulation Value based Verification}

Most of the ADS-based authentication approaches share one weakness for authentication of multi-dimensional range queries: they require to construct one ADS for each possible combination of dimensions in the database. This makes the number of required ADS structures scales exponentially with the number of dimensions. To address this weakness, Papadopoulos et al. [60] integrate the accumulation values with Merkle hash tree (MHT) for the authentication of multidimensional range query evaluation on outsourced databases. They use the authentication protocol of set intersection and difference (Section 4.4.2) as the building block. The authentication method consists of two steps. Let $R$ be the query result. The first step is that the SP computes the set of hash values $R_{i}$ of the tuples that satisfy the query on dimension $i$. It also computes the proof $\pi_{R_{i}}$ that can be used to verify that $R_{i}$ is the set of tuples that match the range constraint on dimension $i$. The second step is that the SP computes $R=\bigcap R_{j}$ and generates the proof $\pi$ of $R$. The client uses the set intersection verification protocol on $\pi$ (Section 4.4.2) to verify the correctness of $R$.

The verification scheme of $[60 \mid$ only supports the verification of range queries. To address the limitation of [60], Zhang et al. [82 proposed a verification system named IntegriDB to accommodate a wide range of SQL queries, including multi-dimensional range queries, JOIN, SUM, MAX/MIN, COUNT, and AVG. The authors designed a sum verification protocol (Section 4.4.3) and a new ADS named Authenticated Interval Tree (AIT-tree), and facilitates them as the key components of IntegriDB. The AIT-tree is designed for key-value pairs. Given a set of key-value pairs $S=\{(k, v)\}$, the AIT-tree is a binary-structured tree constructed from $S$. For each key-value pair $(k, v) \in S$, it corresponds to a leaf node in the AIT-tree, which stores a triplet $(k, v, h)$ with the hash value $h=H(k \| v)$. The leaf nodes are arranged in the order of the keys. The value stored at an internal node $N$ corresponds to the accumulation value (Section 4.4.3) of the leaves in the subtree rooted at $N$. The verification of one-dimensional range query result is similar to [25]. The verification of multi-dimensional range queries and join queries is performed by the authentication protocol of set intersection (Section 4.4.1), by considering the results of each individual dimension/table as a set. Regarding the aggregation queries, they are authenticated by different methods. The SUM queries are verified by using the set summation protocol (Section 4.4.3). In order to facilitate the verification of COUNT operations, the data owner creates an additional attribute $A_{j}^{\prime}$ for each attribute $A_{j}$. The value of $A_{j}^{\prime}$ of the record $t_{i}$ is set as $t_{i}\left[A_{j}^{\prime}\right]=t_{i}\left[A_{j}\right]+1$. The data owner constructs an AIT for the new attribute $A_{j^{\prime}}$. To prove the correctness of $C O U N T\left(A_{j}\right)$, the SP prepares the proof for $S U M\left(A_{j}\right)$ and $S U M\left(A_{j^{\prime}}\right)$, as $\operatorname{COUNT}\left(A_{j}\right)=S U M\left(A_{j^{\prime}}\right)-S U M\left(A_{j}\right)$. MAX/MIN queries can be reduced to single-dimensional range queries. In particular, let $j_{\max }$ be the maximum value for $A_{j}$ that is returned by the SP. The SP prepares the proof for the range query $\sigma_{A_{j} \geq j_{\max }}(D)$. When there are data updates (i.e., insertion/deletion) on the dataset, the data owner updates the AIT-tree by updating the corresponding leaf node $N$ and all the nodes on the path from $N$ to the root of the AIT-tree, as well as the accumulation values of all internal nodes on this path.

\subsection{Trusted Hardware based Verification}

Most of the existing work discussed query verification through software mechanisms. An alternative approach is to rely on some tamper-proof, trusted hardware that is deployed on the untrusted server. Sumeet Bajaj et al. [13] is the only work that we are aware on using trusted hardware for query authentication. They utilize the trusted hardware such as the IBM 4764 [7] co-processor (SCPU) to provide secure execution in an untrusted environment. The main idea is to build MHT based ADS and store the root hash of the ADS inside SCPU. This can eliminate the generation of digital signatures of root node hash, thereby saving a signature operation on each update, and signature verifications for each query. The original query will be rewritten into sub-queries, ensuring that the processing within the SCPU is minimized, and any intermediate results generated by the SP can be validated by the SCPU using the ADS. Any operation executed by the SP that cannot be authenticated should be processed inside the SCPU. [13] provided the query parsing scheme for various types of queries, including range queries, projections, joins, aggregations, grouping and ordering. When there is any data update, it leads to the updates on the MHT based ADS used by the SCPU for verification, which is modified only by the SCPU.

\subsection{Dealing with Data Updates}

For most of the existing deterministic authentication methods, the data owner is responsible to perform all update operations on the data, and update the ADS according to the updates. For the tree-based methods, one way to update the ADS is to first update (insert, delete or modify) the leaf nodes and their hash values. The root signature can be updated by propagating the update on the leaf nodes along their paths up to the root. The updates of both data and ADS are sent back to the SP. In addition, the tree-based approaches require the re-distribution of the digest of ADS root signature to all clients. The naive approach for handling batch updates would be to do all updates to the ADS one by one and update the path from the leaves to the root once per update. Obviously this naive method may lead to expensive cost of performing unnecessary hash function computations on the predecessor path when a large number of updates affect a similar set of nodes (e.g., the same leaf). To optimize the update cost on the ADS, [44] suggests to recompute the hashes of all affected nodes only once, after all the updates have been performed on the tree.

For the signature-based verification methods, any update to a tuple requires to re-compute the signed digests of the tuple as well as its neighboring ones. [54] provides a detailed protocol to support updates on the digests. The accumulation value based authentication methods deal with updates in the similar way. When inserting/deleting a record, the data owner needs to update the accumulation values of these records [82].

When using the trusted hardware, the data owner can issue an update query directly to the SCPU. All updates 
are then performed by the SCPU, and thus eliminating the update overhead at the side of the data owner.

\subsection{Discussion}

One of the major concerns of the proof-based authentication methods is the verification overhead, which includes the size of the proof, the computational overhead in terms of execution time to construct ADS and the VO, and the verification time by the client. The tree-based, signaturebased and accumulation value based authentication methods require one-time setup. At the setup phase, the treebased methods have to construct ADS, while the signaturebased and the accumulation value based methods construct the signatures/accumulation values of individual records. The VO size of the tree-based methods is determined by the size of the query results, which in turn increases both query verification time and data communication overhead. As shown in [20] the communication between the client and SP (e.g., the cloud) can cost up to 3500 picocents/bit, 23 orders of magnitude higher than processing costs (1 US picocent $\left.=\$ 1 \times 10^{14}\right)[13 \mid$. On the other hand, the proof size of the signature-based methods is a constant. Hence, they do not incur the high transmission costs as the tree-based approaches. However, the construction of the signature-based proofs is more expensive than the tree-based approaches, since computing even a single cryptographic trapdoor requires a high number of CPU cycles costing up to 30,000 picocents [13], [20].

\section{Probabilistic Authentication Methods}

Unlike the authentication approaches that use proofs, the authentication without using proofs only can return a probabilistic integrity guarantee, i.e., how likely the returned query results are sound and complete. The existing probabilistic authentication methods can be classified into two types: (1) the checkpoint-based solutions, and (2) the interactive-proof based solutions.

\subsection{Checkpoint-based Solutions}

Sion $\mid 68$ designed the first probabilistic verification approach for query execution on outsourced databases. Given a batch of $b$ queries $\left\{Q_{1}, \ldots, Q_{b}\right\}$, the client first generates $r>1$ random numbers $\left\{x_{1}, \ldots, x_{r}\right\}$, where $1 \leq x_{i} \leq b$. Then, according to the random indexes, the client computes the query results $\left\{\rho\left(Q_{x_{1}}\right), \ldots \rho\left(Q_{x_{r}}\right)\right\}$ for the picked queries. For each query, it generates a challenge token $C\left(Q_{x_{i}}\right)=\left(H\left(\epsilon \| \rho\left(Q_{x_{i}}\right)\right), \epsilon\right)$, where $\epsilon$ is randomly generated. The client sends the query batch as well as the challenge tokens to the SP. The SP returns the query results and the query execution proof $\left\{x_{1}^{\prime}, \ldots, x_{r}^{\prime}\right\}$. The results pass the verification only if $\left\{x_{1}, \ldots, x_{r}\right\}$ matches $\left\{x_{1}^{\prime}, \ldots, x_{r}^{\prime}\right\}$. Due to the one-way non-invertible cryptographic hash function, the SP has to execute the queries over the target dataset to obtain the correct random indexes. The probability that the SP gets all the random indexes by executing $w<b$ queries is

$$
P_{c}(w, r)=\frac{\left(\begin{array}{l}
w \\
r
\end{array}\right)}{\left(\begin{array}{c}
b \\
r
\end{array}\right)} .
$$

The client can control the escape probability that the SP passes the verification to be below a given threshold by adjusting $r$, i.e., the number of queries with challenge tokens. According to the experiment results, an assurance level of $5 \%$ escape probability requires the client to prepare $25 \%$ queries with challenge tokens.

Xie et al. [76] proposed a probabilistic verification framework to check the soundness, completeness and freshness of the query results on outsourced encrypted databases. It is worth noting that the authentication approach in [76] also can protect data privacy as it is applied on the encrypted data. The key idea is that the client creates a small set of fake tuples $\Delta$, and inserts $\Delta$ into the original database $D$. The SP processes queries over the database with $\Delta$, assuming it cannot distinguish real tuples from the fake ones. The correctness of a query result $R_{Q}$ is evaluated by checking if the returned result $R^{S}$ contains $Q(\Delta)$, where $Q(\Delta)$ is the results of evaluating $Q$ on $\Delta$. If it does not, the client believes that the results violates the correctness requirement with $100 \%$ certainty. Otherwise, the client trusts the correctness of $R^{S}$ with a probability. Formally, assume the original dataset $D$ has $N$ tuples, and $K$ fake tuples are inserted into $D$. The probability that an attacker can delete $m$ tuples without being caught is

$$
\prod_{i=0}^{m-1} \frac{N-i}{K+(N-i)}
$$

The empirical study in [76] shows that for a dataset of $N=$ $1,000,000$ tuples, when the fake tuples are more than $10 \%$ of the original data, and more than 50 tuples are deleted, it is close to impossible for the attacker to escape from being caught by the probabilistic approach.

In the reasoning of probabilistic guarantee, the authors only consider tuple deletion since tuple modification or insertion can be easily detected through the following data authentication. Before outsourcing, the data owner inserts a special digest value for each (real/fake) tuple. For any tuple $t=\left\{t i d, a_{1}, \ldots, a_{m}\right\}$, where $t i d$ is the unique tuple identifier, the digest is calculated as

$$
h(t)= \begin{cases}H\left(t i d \oplus a_{1} \oplus \cdots \oplus a_{m}\right) & \text { if } t \text { is real } \\ H\left(t i d \oplus a_{1} \oplus \cdots \oplus a_{m}\right)+1 & \text { if } t \text { is fake }\end{cases}
$$

On receiving any tuple $t$ from the $\mathrm{SP}$, the client can tell if it is a real or fake tuple from the digest value. The way to generate digest values facilitates efficient data update operation, as the digest of new tuples can be readily calculated at the client side.

Di Vimercati et al. [24], |26] applied the probabilistic authentication method to join queries. The outsourcing framework considers the join of two relations, $B_{l}$ and $B_{r}$ hosted at two storage SPs $S_{l}$ and $S_{r}$. The integrity guarantee of $B_{l} \bowtie B_{r}$ is verified by using three types of fake tuples inserted into the outsourced dataset: (1) markers tuples inserted to both $B_{l}$ and $B_{r}$; (2) twins tuples, which are a portion of real records that each of the storage SPs duplicates locally before sending it to the SP; (3) salts/buckets tuples that are inserted to destroy recognizable frequencies of combinations in one-to-many joins. Salts are used on the tuples at the many-side of the join so that occurrences of a same value become distinct. Meanwhile the salted replicas are created 
at one-side of the join to create the corresponding matching. Bucketization allows multiple occurrences of the same (encrypted) value at the many-side of the join, requiring that all the values have the same number of occurrences. The client can verify the result correctness regarding the markers and twins. The probability that no marker is omitted is $\left(1-\frac{o}{f}\right)^{m}$, where $f$ is the cardinality of a relation with $t$ twin pairs and $m$ markers, and $o$ is the number of original tuples that are omitted without being detected. The probability that for each twin pair, either both tuples are omitted or both are preserved is $\left(1-2 \frac{o}{f}+2\left(\frac{o}{f}\right)^{2}\right)^{t}$.

\subsection{Interactive Proof based Verification}

Yupeng Zhang et al. [81] designed $v S Q L$, a system for verifiable SQL queries over dynamic outsourced databases. vSQL combines two different approaches, namely informationtheoretic interactive proof system [23] and a novel scheme for verifiable polynomial delegation that can provide auxiliary inputs for the proof. vSQL first translates SQL queries as arithmetic circuits. Then it relies on an informationtheoretic interactive proof system named the CMT protocol [23], which allows a client to verify that $y=C(x)$, where $x$ is the clients data, and $C$ is a circuit corresponding to the clients query. However, it is not feasible to apply the CMT protocol directly since the client may not be able to store the data $x$ (i.e., the input database). To address this problem, vSQL designs a new polynomial-delegation protocol, which allows the client to be able to evaluate a certain multivariate polynomial $p_{x}$ that depends on $x$ (but not on $C$ ) at a random point. In particular, the data owner treats the database $D$ as an array of $|D|$ elements, and computes the multi-linear extension $D^{\prime}$ of $D$. The data owner uses the polynomialdelegation protocol to generate the commitment com of $D^{\prime}$, and sends com to the client and sends $D$ to the SP. At the query evaluation phase, the client verifies the query result following the $C M T$ protocol. The verification of all layers except the last one follows the CMT protocol between the client and the SP. In the last step of the CMT protocol, it has to evaluate at a random point of $D^{\prime}$ that is picked by the SP. Since the client does not have access to $D^{\prime}$, it verifies the last step by using the commitment com via the polynomialdelegation protocol. Since the point of the last step is not the same as used for all previous steps, vSQL only provides a non-deterministic guarantee of the query results. If the result returned by the SP is incorrect, the client will reject it with overwhelming probability.

\subsection{Dealing with Data Updates}

The checkpoint-based methods can deal with data updates efficiently. The data owner can choose new checkpoints in the updated records. The digest values of these new checkpoint records can be readily calculated at the client side. The interactive-proof based method [81] supports efficient data updates by separating the computation of the update from its verification. The SP has to update the digest $\mathrm{com}$. The client can provably evaluate if com is updated by using the polynomial-delegation scheme.

\subsection{Discussion}

The existing checkpoint-based approaches have different assumptions of the SP. [68] requires that the SP to be aware of the verification protocol. |76| relaxes the assumption and makes the verification approach to be transparent to the SP. The SP is not required to be involved in the verification protocol at all. This reduces the computational overhead by the SP. According to the empirical study of $|76|$, the fake tuple based approach [76] incurs much cheaper verification setup overhead at the data owner side than the challenge token based approach $|68|$ and MHT tree based deterministic approach [57]. However, the computational cost at the SP side by the fake tuple based approach $\mid 76]$ can be higher than the two aforementioned approaches, due to the large number of inserted fake tuples to achieve high probabilistic guarantee.

The alternative interactive proof based approach 81 aims to make the expensive theoretical interactive proof system to be practical. Compared with the generic verification approaches [17], [33], it leads to a significant performance speedup at the SP side. Its performance is also comparable to a deterministic approach [82] that only supports a restricted subclass of SQL queries.

\section{Freshness Authentication}

Most of the works discussed so far only consider the verification of soundness and completeness of the query results. In this section, we review the existing studies on verification of freshness of the query results, i.e., these results are obtained by executing the queries over the most up-to-date data. Similar to the verification of soundness and completeness, the freshness verification methods can be classified into deterministic and probabilistic approaches.

Deterministic approaches. A straightforward solution is to extend the proof-based solutions to provide freshness verification. The key challenge of such extension is to ensure that the signatures attached to individual tuples are indeed constructed from the most up-to-date data. Then the client can verify the freshness of the query results from the constructed proof of the query results in the similar way as the existing proof-based methods, as long as the proof is constructed from the up-to-date signatures, for example, either by aggregated signature or by the tree-based ADS. $\mathrm{Li}$ et al. |44| first raises the issue of query freshness. They provide a number of solutions, including publishing a list of revoked signatures, including the time interval of validity as part of the signed message and reissuing the signature after the interval expires, and using hash chains to confirm validity of signatures at frequent intervals. They point out that any of these approaches can be applied directly on the tree-based solutions to update the single signature of the root of the tree. Each data update will require reissuing one signature only. Xie et al. [77] designs a scheme that associates the signature $S_{t}$ (e.g., the aggregated signature or the root signature of MHT tree) with a certificate Certificate $_{C_{A}}\left(S_{t}, t\right)$ in the outsourced database, to indicate that the current signature at time $t$ is $S_{t}$. When a client retrieves the query result, it also retrieves the signature and verifies if it is for timestamp $t$. Pang et al. [59] combined signature chaining with timestamps. The key idea is that the timestamps are embraced into the calculation of hash values of tuples. Thus the signature of a tuple $t_{i}$ is calculated as $\sigma_{i}=\operatorname{sign}\left(H\left(i|| t_{i}|| t_{i-1} \| t_{i} . t s\right)\right)$, where $\operatorname{sign}()$ is a signature 
function (e.g. RSA [66|), $H()$ is a collision-free hash function, and $t_{i-1}$ is the immediate left neighbor of $t_{i}$ along an ordering attribute, and $t s$ denotes the timestamp of the last update for tuple $t_{i}$. Based on the this signature scheme, the proof is constructed in the similar way as [53]. The proof can be used to verify authenticity, soundness, completeness, and freshness.

Probabilistic approaches. The key challenges to extend the checkpoint-based authentication methods to support freshness verification are as follows. First, the client must know what are the most up-to-date fake tuples in the outsourced database. This means that the client must be aware of all insertions and deletions of the fake tuples starting from the beginning. Second, it is important to ensure the fake tuple based scheme is provably secure, i.e., the attacker cannot distinguish fake operations from real operations. To address these challenges, [77] devise a mechanism to make all fake operations deterministic so that the client can derive the latest status of the fake tuples in the outsourced database. Specifically, the fake operations scheme is defined as $(F S, T, H)$, where $F S$ is a set of functions to generate the fake tuples, $T$ is a function to decide when to submit the fake operations and $H$ is a function to decide when a function in $F S$ is used to create fake tuples. The paper shows that the scheme is a provably secure scheme whose security can be reduced to the underlying encryption primitives.

\section{Query Authentication With Other Secu- RITY FEATURES}

Other security goals can be considered in parallel with query authentication in the DaaS framework. For example, how to efficiently authenticate query results without violating access control policies, and how to verify the integrity of results on privacy-preserving query evaluation. In this section, we review the existing works that integrate query integrity authentication with other security features.

\subsection{Integrity and Privacy}

Sarvjeet Singh et al. [67] consider a two-party scenario, in which the data owner has a private database, and the external querier would like to obtain some information from the private database by sending the queries to the data owner. The external querier desires to obtain a proof that shows the results returned by the database owner indeed reflect the correct evaluation of the submitted query over an uncorrupted version of the database. On the other hand, the data owner does not reveal any private information to the querier except the query results. The goal is to design a query authentication method for private databases, aiming to minimize the amounts of data revealed to the untrusted third party, except the query results. The paper included several solutions with various degrees of privacy, computational complexity and data bandwidth. The recommended solution is to use Merkle tree for query authentication. The key idea is that the data owner constructs a Merkle tree, and sends the query results with a proof constructed from the Merkle tree. This can preserve database privacy as it reveals only the hashes of tuples that are not part of the result. The integrity of query results is verified by reconstructing the root signature of the hash tree, similar to [25].
Wang et al. |73| consider a three-party scenario that contains the data owner, the SP, and the client who is not necessarily to be the data owner. To protect the private data from the untrusted SP, the data is encrypted by the methods that support encrypted queries over encrypted databases. To provide query verification guarantee, they design a novel encryption method called Dual Encryption. The key idea of dual encryption is to encrypt the entire data $D$ using a primary encryption key $k$, and encrypt a selected, small subset of $D$ using a secondary encryption key $k^{\prime}$. The two encrypted datasets are merged and stored at the SP as a single dataset. The purpose of dual encryption is to allow for sophisticated cross examination on query results. Assume a client has a batch of queries $Q=\left\{q_{1}, \ldots, q_{u}\right\}$. For each query $q_{i} \in Q$, the client sends its encrypted query $q_{i}^{k}$ to the SP and gets its result $\rho\left(q_{i}^{k}\right)$. To verify the integrity of $\rho\left(q_{i}^{k}\right)$, it sends $q_{i}^{k^{\prime}}$ to the SP, where $q_{i}^{k}$ and $q_{i}^{k^{\prime}}$ are semantically identical (i.e., they return the same set of records). The client analyzes both $\rho\left(q_{i}^{k}\right)$ and $\rho\left(q_{i}^{k^{\prime}}\right)$ by checking if for each replicated tuple $t \in \rho\left(q_{i}^{k}\right), t$ also appears in $\rho\left(q_{i}^{k^{\prime}}\right)$. To help identify how the tuples are encrypted, the data owner attaches dual information to each record, which is generated by secret key and one-way hashing. Regarding the security guarantee of the dual encryption schemes, the authors investigated two possible attacks, namely the query correspondence attack and the distribution attack. The query correspondence attack considers the case that if an adversary can find out that two encrypted queries $\rho\left(q_{i}^{k}\right)$ and $\rho\left(q_{i}^{k^{\prime}}\right)$ are indeed semantically identical, it can break the verification. To defend against this attack, the dual encryption scheme is revised by requiring that $\rho\left(q_{i}^{k}\right)$ and $\rho\left(q_{i}^{k^{\prime}}\right)$ should not be identical but indeed have certain overlap. The distribution attack considers an adversary who may discover data correspondence with high probability by studying the distribution of query results. The solution to defend against this attack is to issue the checking query based on the answer size of the query to be verified. Only when the answer size is large enough the checking query can be issued.

\subsection{Integrity and Data Confidentiality}

Pang et al. [57] is the first work that considers query authentication with the respect of the access control policy. They consider the completeness verification for selectionprojection queries, with the assumption that the original selection-projection query has been re-written as a new selection-projection query. The challenge is to make sure that during the construction of completeness proof, the left and right boundaries of the query results that are used for VO do not violate the access control policy. To address this challenge, the data owner inserts two fictitious entries, a left delimiter $r_{0} \in(L, U)$ and a right delimiter $r_{n+1} \in(L, U)$ into $D$. The two fictitious entries ensures that the boundary records used in the proof do not violate the access control policy. For completeness verification, the digital signature of the real tuples in the query results are constructed. While for the two delimiters, their signatures are constructed by aggregating with the boundary records. Formally,

$$
\begin{aligned}
\operatorname{sig}\left(r_{0}\right) & =s\left(h\left(h(L)\left|g\left(r_{0}\right)\right| g\left(r_{1}\right)\right)\right), \\
\operatorname{sig}\left(r_{n+1}\right) & =s\left(h\left(g\left(r_{n}\right)\left|g\left(r_{n+1}\right)\right| h(U)\right)\right) .
\end{aligned}
$$


Based on the signatures, the completeness verification is performed in the similar way as in [58].

Kundu et al. [43] consider the scenario that the data organization structures encode sensitive information (such as in XML documents). The goal is to provide both integrity and confidentiality not only for the content, but also for the structure. The concept of confidentiality is interpreted as requiring a user to receive only those nodes and the structural information that the user is allowed to access, according to the stated access control policies. A user should not receive nor should be able to infer any information about the content and presence of nodes and structural information that the user is not authorized to access. Based on this, [43] formally defined the concept of structure confidentiality. The aim is to enable an user to authenticate a subtree $S$ from a tree $T$ without leaking any other nodes from $T S$ ( refers to the cut operation). They designed a novel concept called structural signature, which is based on the structure of the tree defined by tree traversals - postorder, pre-order and in-order. In particular, the structural signature of a node $x$ in tree $T$ consists of a hash of the structural position, the content of $x$, and the (salted) signature of the tree, where the structural position of a node is defined as a pair of randomized post-order (RPON) and randomized pre-order (RRON). To share the tree with a third-party that has the authorization to access the subtree $S_{z}, S_{z}$ can be shared according to two different strategies: (1) by sharing the signed subtree - its nodes and the structure or (2) by sharing the signed nodes in the subtree and letting the third-party reconstruct the subtree using the RPONs and RRONs of the nodes. The authors considered both options for integrity verification, with the same goal as building the correct signature of the tree to show correctness. They provide the security analysis which shows the structural signatures do not lead to any leakage of (1) node signatures, and information about the (2) existence of nodes, (3) structural relations or (4) structural order among nodes.

\section{Conclusion AND Future Work}

In this survey, we reviewed the existing studies on integrity authentication of SQL query evaluation for the DaaS paradigm. We categorize these works by how the verification is performed and the integrity guarantee that these works can achieve. There exists the trade-off between the integrity guarantee and the performance of the verification methods. The deterministic integrity guarantee can be achieved but with possibly expensive setup cost and verification overhead; such overhead can be improved by relaxing the integrity guarantee to be probabilistic.

In the rest of this section, we discuss several interesting directions for the future work.

\subsection{Authentication of Query Evaluation on Big Data}

The three well-known properties of Big data is $3 \mathrm{Vs}$ (volume, variety and velocity). Volume refers to the amount of data, variety refers to the number of types of data and velocity refers to the speed of data processing. The three properties raise different challenges to the authentication of query evaluation. The big data volume requires that the authentication methods must be scalable; the setup and verification should not add significant overhead on query evaluation itself. The fast velocity requires that the authentication methods must support frequent updates efficiently. The large variety requires that the authentication methods should be able to support various data types. A naive method is to construct an ADS for each data type, and thus a proof for each data type too. But this may bring overwhelming verification overhead. An interesting research direction that worths to explore is how to address the challenges of volume, variety and velocity of Big data for the design of efficient and scalable authentication solutions.

MapReduce has emerged as an important computational model for data intensive applications. The MapReduce infrastructure consists of two primitives: (a) a map function that distributes the input to multiple mapper nodes to generate intermediate key-values in parallel, and (b) a reduce function that allows multiple reducer nodes to merge the intermediate pairs associated with the same key and then to generate the final outputs. Intuitively, all mappers and reducers can be compromised and return wrong intermediate and/or final results. Therefore, cloud-based query evaluation raises two main challenge: (1) how to authenticate the query results in the format of key-value pairs; and (2) how to authenticate both the intermediate and final results for MapReduce execution. A few papers have considered integrity verification of MapReduce execution [34], [71], [74]. Intuitively, the intermediate results can be authenticated by applying the existing solutions on each mapper. However, this requires to construct an ADS independently for each mapper, which can be extremely expensive. The main challenge is how to address the verification overhead issue for the query results in a distributed fashion. In particular, how to construct the ADS to support efficient verification of both intermediate query results by the mappers and final query results by the reducers.

\subsection{Authentication for Various Outsourcing Paradigms}

In general, the outsourcing paradigms can be classified into three types:

- Infrastructure-as-a-Service (IaaS) paradigm: the data owner can deploy and run arbitrary software including operating systems and applications. The SP provides the computing infrastructure, e.g., data storage and hardware for the computation. A typical IaaS example is Amazon EC2 Web Service [1].

- Platform-as-a-Service (PaaS) paradigm: The SP delivers a computing platform, typically including operating system, execution environment, database, and web server. Some well-known PaaS offers include Microsoft Azure [10] and Google Cloud SQL [4].

- Software-as-a-Service (SaaS) paradigm: The clients use the SP's applications running on an outsourced infrastructure (e.g., the cloud). The applications are accessible from various client devices through either a thin client interface, such as a web browser (e.g., web-based email), or a program interface.

Different computational paradigms bring different challenges of authentication. For example, compared with the IaaS paradigm, the SP of Paas and SaaS paradigms has more cheating power on the computations, as the client has 
neither knowledge of the computations nor how these computations are configured and executed. Therefore, the verification of result integrity for the SaaS and PaaS paradigms is more challenging, especially for those computations whose outputs highly depend on the setup of the computations (e.g., the initial centroids of $k$-means clustering [46]).

\subsection{Authentication of Various Types of Outsourced Computations}

This survey focuses on SQL query evaluation only. In practice, the outsourcing paradigm provides a broader range of data analytics services, ranging from simple aggregation to Web search to sophisticated data mining and learning, to name a few. Below we present a few examples of outsourced computations, and discuss the challenges and potential research directions for the verification of these types of outsourced computations.

Keyword search on Web. Web search engines are typical outsourced computations. The search service providers may return incorrect search results due to various reasons (e.g., to make profits from advertisements) |35|. Furthermore, the service providers may return the results in a wrong ranking (e.g., to promote some websites). Therefore, the authentication goals are two-fold: (1) to verify that the returned keyword search results are sound, complete, and fresh, and (2) to verify the ranking of the returned results is accurate. [35], [55] have initiated the research on authentication of soundness, completeness, and freshness of the Web-search content. How to verify the ranking of the results is still unsolved. Verification of top-k query evaluation has been studied under different contexts, e.g., for sensor networks [80] and location-based services [19]. It will be interesting to explore if these top-k query authentication methods can be adapted to keyword search queries.

Outsourced data mining and machine learning. Big corporations like Amazon, Google and Microsoft are providing cloud-based data mining and learning services in various forms. Amazon Web Services (AWS) provides computation capacity and data storages via Amazon Elastic Compute Cloud (EC2) [1] and Simple Storage Service (S3) [2]. Google provides cloud machine learning engines [3] for various machine learning applications, including customer purchase prediction and spam detection, to name a few. Microsoft provides big data analytics services on Windows Azure cloud [10]. This raises the issue of how to evaluate the result integrity of these outsourced machine learning computations. There are several interesting research questions. First, can any existing ADS structure (e.g. MB-tree [44] and VB-tree [58|) be adapted to the authentication solutions for outsourced machine learning and data mining computations? Second, given the high complexity of machine learning computations, the deterministic approach may not be appropriate as it can bring expensive overhead. On the other hand, it is not straightforward how to adapt the existing probabilistic approaches |76| to the outsourced machine learning computations, since inserting some fake tuples can change the machine learning results (e.g., classification and clustering) of the original data. The challenge is how to design those fake tuples for authentication while keeping the original data analytics results unchanged. The existing authentication solutions include various types of data mining computations, including association rule mining [27], [28], [29], outlier mining [30], [48], clustering [46], Bayesian network construction [47], and collaborative filtering [70], [72]. Many of these solutions (e.g. [46], [47], [48]) consider the probabilistic approaches. One of the weaknesses of these approaches is that they may be vulnerable against the attacker who is aware of the authentication mechanism, in particular, how the fake data points are constructed. Then the attacker can distinguish the fake data points from the real ones. An interesting research direction is to design robust probabilistic verification methods that can address the trade-off between efficiency and the robustness of the authentication methods.

Spatial query evaluation. A large body of work has considered query evaluation on spatial databases (e.g. [39], [40], [41], [62], [79]). The goal of authentication is to verify if the returned locations satisfy the given constraints (e.g. within a range, one of the nearest neighbor, etc.). The main challenge of spatial query authentication is how to compute the distances efficiently for verification. Various techniques (e.g., Voronoi diagram and R-trees) are used to represent the underlying spatial data set. The remaining technical issues to be resolved is how to design efficient, online authentication methods that can deal with dynamic location data that are updated frequently. This requires that the authentication methods can support fast updates on the ADS, quick construction of the proof of the query results, and cheap verification at the client side who may use resource-constrained devices such as mobile phones for verification.

\section{ACKNOWLEDGEMENTS}

This material is based upon work supported by the National Science Foundation (NSF) under Grant No. 1350324 and 1464800.

\section{REFERENCES}

[1] Amazon elastic compute cloud (ec2) http://aws.amazon.com/ec2/.

[2] Amazon simple storage service http://aws.amazon.com/s3/.

[3] Google cloud machine https://cloud.google.com/ml-engine/.

[4] Google cloud sql. https://cloud.google.com/sql/.

[5] Has amazon gone off the deep end with cloud pricing complexity. http://www.linkedin.com/groups/Has- Amazon-gone-off-deep2931633.S.180931629.

[6] Hidden charges when using amazon aws' free tier. http://mhlakhani.com/blog/2011/01/hidden-charges-awsfree-tier $/$.

[7] Ibm 4764 pci-x, 4764 pcie cryptographic coprocessors. http://www-03.ibm.com/security/crytocards/.

[8] Microsoft cloud database https://azure.microsoft.com/en-us/services/sql-database/.

[9] The search for extraterrestrial intelligence project. university of california, berkeley. http://setiathome.berkeley.edu/.

[10] Windows azure cloud. http://www.windowsazure.com/en-us/.

[11] Daniel J. Abadi. Data management in the cloud: Limitations and opportunities. In IEEE Data Engineering Bulletin, 32(1):3-12, 2009.

[12] Giuseppe Ateniese, Seny Kamara, Jonathan Katz, et al. Proofs of storage from homomorphic identification protocols. In Proceedings of Annual International Conference on the Theory and Applications of Cryptology and Information Security (Asiacrypt), volume 9, pages 319-333, 2009. 
[13] Sumeet Bajaj and Radu Sion. Correctdb: Sql engine with practical query authentication. Proceedings of the VLDB Endowment, 6(7):529540, 2013.

[14] Niko Barić and Birgit Pfitzmann. Collision-free accumulators and fail-stop signature schemes without trees. In Proceedings of the International Conference on Theory and Application of Cryptographic Techniques (Eurocrypt), pages 480-494, 1997.

[15] Josh Benaloh and Michael De Mare. One-way accumulators: A decentralized alternative to digital signatures. In Workshop on the Theory and Application of of Cryptographic Techniques (Eurocrypt), pages 274-285, 1993.

[16] Jon Louis Bentley. Multidimensional binary search trees used for associative searching. Communications of the ACM, 18(9):509-517, 1975.

[17] Nir Bitansky, Ran Canetti, Alessandro Chiesa, and Eran Tromer. From extractable collision resistance to succinct non-interactive arguments of knowledge, and back again. In Proceedings of the $3 \mathrm{rd}$ Innovations in Theoretical Computer Science Conference, pages 326349, 2012.

[18] Dan Boneh, Craig Gentry, Ben Lynn, and Hovav Shacham. Aggregate and verifiably encrypted signatures from bilinear maps. In Proceedings of International Conference on Theory and Application of Cryptographic Techniques (Eurocrypt), volume 2656, pages 416-432, 2003.

[19] Qian Chen, Haibo Hu, and Jianliang Xu. Authenticating top-k queries in location-based services with confidentiality. Proceedings of the VLDB Endowment, 7(1):49-60, 2013.

[20] Yao Chen and Radu Sion. To cloud or not to cloud?: musings on costs and viability. In Proceedings of the 2nd ACM Symposium on Cloud Computing, page 29, 2011.

[21] Weiwei Cheng, HweeHwa Pang, and Kian-Lee Tan. Authenticating multi-dimensional query results in data publishing. In Proceedings of the 20th Annual IFIP Conference of Data and Applications Security (DBSec), pages 60-73, 2006.

[22] Thomas Claburn. Yahoo scales its web analytics database to petabyte range. http://www.dbms2.com/2008/05/29/yahooscales-web-analytics-database-petabyte/.

[23] Graham Cormode, Michael Mitzenmacher, and Justin Thaler. Practical verified computation with streaming interactive proofs. In Proceedings of the 3rd Innovations in Theoretical Computer Science Conference, pages 90-112, 2012.

[24] Sabrina De Capitani di Vimercati, Sara Foresti, Sushil Jajodia, Stefano Paraboschi, and Pierangela Samarati. Efficient integrity checks for join queries in the cloud. Journal of Computer Security, 24(3):347-378, 2016.

[25] Premkumar Devanbu, Michael Gertz, Charles Martel, and Stuart G Stubblebine. Authentic data publication over the internet. Journal of Computer Security, 11(3):291-314, 2003.

[26] Sabrina De Capitani di Vimercati, Sara Foresti, Sushil Jajodia, Stefano Paraboschi, and Pierangela Samarati. Optimizing integrity checks for join queries in the cloud. In Proceedings of the IFIP Annual Conference on Data and Applications Security and Privacy (DBSec), pages 33-48, 2014.

[27] Boxiang Dong, Ruilin Liu, and Hui Wendy Wang. Result integrity verification of outsourced frequent itemset mining. In Proceedings of IFIP Annual Conference on Data and Applications Security and Privacy (DBSec), pages 258-265. 2013.

[28] Boxiang Dong, Ruilin Liu, and Hui Wendy Wang. Trust-butverify: Verifying result correctness of outsourced frequent itemset mining in data-mining-as-a-service paradigm. IEEE Transactions on Services Computing, 9(1):18-32, 2016.

[29] Boxiang Dong, Ruilin Liu, and Wendy Hui Wang. Integrity verification of outsourced frequent itemset mining with deterministic guarantee. In Proceedings of the IEEE International Conference on Data Mining (ICDM), pages 1025-1030, 2013.

[30] Boxiang Dong, Hui (Wendy) Wang, Anna Monreale, Dino Pedreschi, Fosca Giannotti, and Wenge Guo. Authenticated outlier mining for outsourced databases. IEEE Transactions on Dependable and Secure Computing, 2017.

[31] D Eastlake 3rd and Paul Jones. Us secure hash algorithm 1 (sha1). Technical report, 2001.

[32] Rosario Gennaro, Craig Gentry, and Bryan Parno. Non-interactive verifiable computing: Outsourcing computation to untrusted workers. Proceedings of the Advances in Cryptology (CRYPTO), pages 465-482, 2010.

[33] Rosario Gennaro, Craig Gentry, Bryan Parno, and Mariana Raykova. Quadratic span programs and succinct nizks without pcps. In Annual International Conference on the Theory and Applications of Cryptographic Techniques, pages 626-645, 2013.

[34] Esha Ghosh, Olga Ohrimenko, and Roberto Tamassia. Efficient verifiable range and closest point queries in zero-knowledge. Proceedings of Privacy Enhancing Technologies, 2016(4):373-388, 2016.

[35] Michael T Goodrich, Charalampos Papamanthou, Duy Nguyen, Roberto Tamassia, Cristina Videira Lopes, Olga Ohrimenko, and Nikos Triandopoulos. Efficient verification of web-content searching through authenticated web crawlers. Proceedings of the Very Large Data Base Endowment (VLDB Endowment), 5(10):920-931, 2012.

[36] Antonin Guttman. R-trees: a dynamic index structure for spatial searching. In Proceedings of the ACM Special Interest Group on Management of Data ACM (SIGMOD) international conference on Management of data, 1984.

[37] H. Hacigumus, B. Iyer, and S. Mehrotra. Providing database as a service. In Proceedings 18th International Conference on Data Engineering, pages 29-38, 2002.

[38] Ching-Tien Ho, Rakesh Agrawal, Nimrod Megiddo, and Ramakrishnan Srikant. Range queries in OLAP data cubes, volume 26. 1997.

[39] Haibo Hu, Qian Chen, and Jianliang Xu. Verdict: Privacypreserving authentication of range queries in location-based services. In Proceedings of the IEEE 29th International Conference on Data Engineering (ICDE), pages 1312-1315, 2013.

[40] Ling $\mathrm{Hu}$, Wei-Shinn $\mathrm{Ku}$, Spiridon Bakiras, and Cyrus Shahabi. Spatial query integrity with voronoi neighbors. IEEE Transactions on Knowledge and Data Engineering (TKDE), 25(4):863-876, 2013.

[41] Yinan Jing, Ling $\mathrm{Hu}$, Wei-Shinn $\mathrm{Ku}$, and Cyrus Shahabi. Authentication of $\mathrm{k}$ nearest neighbor query on road networks. IEEE Transactions on Knowledge and Data Engineering (TKDE), 26(6):14941506, 2014.

[42] L. Kahney. Cheaters bow to peer pressure. Wired Magazine, Feb. 15, 2001.

[43] Ashish Kundu and Elisa Bertino. Structural signatures for tree data structures. Proceedings of the Very Large Database Endowment (VLDB), 1(1):138-150, 2008.

[44] Feifei Li, Marios Hadjieleftheriou, George Kollios, and Leonid Reyzin. Dynamic authenticated index structures for outsourced databases. In Proceedings of the International Conference on Management of Data, pages 121-132, 2006.

[45] Feifei Li, Marios Hadjieleftheriou, George Kollios, and Leonid Reyzin. Authenticated index structures for aggregation queries. ACM Transactions on Information and System Security (TISSEC), page 32, 2010.

[46] Ruilin Liu, Hui Wang, Philippos Mordohai, and Hui Xiong. Integrity verification of k-means clustering outsourced to infrastructure as a service (iaas) providers. In Proceedings of the SIAM International Conference on Data Mining (ICDM), pages 632-640, 2013.

[47] Ruilin Liu, Hui Wang, and Changhe Yuan. Result integrity verification of outsourced bayesian network structure learning. In Proceedings of the SIAM International Conference on Data Mining (ICDM), pages 713-721, 2014.

[48] Ruilin Liu, Hui Wendy Wang, Anna Monreale, Dino Pedreschi, Fosca Giannotti, and Wenge Guo. Audio: An integrity auditing framework of outlier-mining-as-a-service systems. In Proceedings of the Joint European Conference on Machine Learning and Knowledge Discovery in Databases (ECML-PKDD), pages 1-18, 2012.

[49] Alfred Menezes, Scott Vanstone, and Tatsuaki Okamoto. Reducing elliptic curve logarithms to logarithms in a finite field. In Proceedings of the twenty-third annual ACM symposium on Theory of computing (STOC), pages 80-89, 1991.

[50] Curt Monash. The 1-petabyte barrier is crumbling http://www.networkworld.com/community/node/31439.

[51] Kyriakos Mouratidis, Dimitris Sacharidis, and Hweehwa Pang. Partially materialized digest scheme: An efficient verification method for outsourced databases. The Very Large Database (VLDB) Journal, 18(1):363-381, 2009.

[52] Einar Mykletun, Maithili Narasimha, and Gene Tsudik. Authentication and integrity in outsourced databases. ACM Transactions on Storage (TOS), 2(2):107-138, 2006.

[53] Maithili Narasimha and Gene Tsudik. Authentication of Outsourced Databases Using Signature Aggregation and Chaining, pages 420-436. 2006.

[54] Maithili Narasimha and Gene Tsudik. Authentication of outsourced databases using signature aggregation and chaining. In 
Proceedings of the International Conference on Database Systems for Advanced Applications, pages 420-436, 2006.

[55] Duy Nguyen. Verification of Web-Content Searching Through Authenticated Web Crawler. PhD thesis, thesis submitted to Department of Computer Science Brown University.

[56] Glen Nuckolls. Verified query results from hybrid authentication trees. In Proceedings of Data and Applications Security XIX, 19th Annual IFIP WG 11.3 Working Conference on Data and Applications Security (DBSec), pages 84-98, 2005.

[57] HweeHwa Pang, Arpit Jain, Krithi Ramamritham, and Kian-Lee Tan. Verifying completeness of relational query results in data publishing. In Proceedings of the ACM International Conference of Special Interest Group on Management of Data (SIGMOD), pages 407$418,2005$.

[58] HweeHwa Pang and K-L Tan. Authenticating query results in edge computing. In Proceedings of the International Conference on Data Engineering (ICDE), pages 560-571, 2004.

[59] HweeHwa Pang, Jilian Zhang, and Kyriakos Mouratidis. Scalable verification for outsourced dynamic databases. Proceedings of the Very Large Database (VLDB) Endowment., 2(1):802-813, 2009.

[60] Dimitrios Papadopoulos, Stavros Papadopoulos, and Nikos Triandopoulos. Taking authenticated range queries to arbitrary dimensions. In Proceedings of the Conference on Computer and Communications Security (CCS), pages 819-830, 2014.

[61] S. Papadopoulos, D. Papadias, W. Cheng, and K. L. Tan. Separating authentication from query execution in outsourced databases. In Proceedings of the IEEE 25th International Conference on Data Engineering (ICDE), pages 1148-1151, 2009.

[62] Stavros Papadopoulos, Lixing Wang, Yin Yang, Dimitris Papadias, and Panagiotis Karras. Authenticated multistep nearest neighbor search. IEEE Transactions on Knowledge and Data Engineering (TKDE), 23(5):641-654, 2011.

[63] Charalampos Papamanthou, Roberto Tamassia, and Nikos Triandopoulos. Optimal verification of operations on dynamic sets. In Proceedings of the Annual Cryptology Conference (CRYPTO), pages 91-110, 2011.

[64] Bryan Parno, Jon Howell, Craig Gentry, and Mariana Raykova. Pinocchio: Nearly practical verifiable computation. In Proceedings of the IEEE Symposium on Security and Privacy (SEP), pages 238252, 2013.

[65] R.C.Merkle. Protocols for public key cryptosystems. In Symposium on Security and Privacy (SEP), 1980.

[66] Ronald L Rivest, Adi Shamir, and Leonard Adleman. A method for obtaining digital signatures and public-key cryptosystems. Communications of the ACM, 21(2):120-126, 1978.

[67] Sarvjeet Singh and Sunil Prabhakar. Ensuring correctness over untrusted private database. In Proceedings of the 11th International Conference on Extending Database Technology: Advances in Database Technology (EDBT), pages 476-486, 2008.

[68] Radu Sion. Query execution assurance for outsourced databases. In Proceedings of the 31st international conference on Very Large Data bases (VLDB), pages 601-612, 2005.

[69] Douglas R Stinson. Cryptography: theory and practice. CRC press, 2005.

[70] Qiang Tang, Balázs Pejó, and Husen Wang. Protect both integrity and confidentiality in outsourcing collaborative filtering computations. In Proceedings of the International Conference on Cloud Computing (CLOUD), pages 941-946, 2016.

[71] H. Ulusoy, M. Kantarcioglu, and E. Pattuk. Trustmr: Computation integrity assurance system for mapreduce. In Proceedings of IEEE International Conference on Big Data (Big Data), pages 441-450, 2015.

[72] Jaideep Vaidya, Ibrahim Yakut, and Anirban Basu. Efficient integrity verification for outsourced collaborative filtering. In Proceedings of the International Conference on Data Mining (ICDM), pages 560-569, 2014.

[73] Haixun Wang, Jian Yin, Chang-shing Perng, and Philip S Yu. Dual encryption for query integrity assurance. In Proceedings of the 17th ACM conference on Information and knowledge management (CIKM), pages $863-872,2008$.

[74] Y. Wang and J. Wei. Viaf: Verification-based integrity assurance framework for mapreduce. In Proceedings of the IEEE 4th International Conference on Cloud Computing (CLOUD), pages 300-307, 2011.

[75] D. E. Weisman and C. Buss. Database functionality high, analytics lags. In Forrester Brief: Business Technographics North America, 2001.
[76] Min Xie, Haixun Wang, Jian Yin, and Xiaofeng Meng. Integrity auditing of outsourced data. In Proceedings of the 33rd international conference on Very Large Data bases (VLDB), pages 782-793, 2007.

[77] Min Xie, Haixun Wang, Jian Yin, and Xiaofeng Meng. Providing freshness guarantees for outsourced databases. In Proceedings of the 11th International Conference on Extending Database Technology: Advances in Database Technology (EDBT), pages 323-332, 2008.

[78] Yin Yang, Dimitris Papadias, Stavros Papadopoulos, and Panos Kalnis. Authenticated join processing in outsourced databases. In Proceedings of the International Conference on Management of data (SIGMOD), pages 5-18, 2009.

[79] Duncan Yung, Eric Lo, and Man Lung Yiu. Authentication of moving range queries. In Proceedings of the 21st ACM international conference on Information and knowledge management (CIKM), pages 1372-1381, 2012.

[80] Rui Zhang, Jing Shi, Yunzhong Liu, and Yanchao Zhang. Verifiable fine-grained top-k queries in tiered sensor networks. In Proceedings of IEEE INFOCOM conference, pages 1-9, 2010.

[81] Y. Zhang, D. Genkin, J. Katz, D. Papadopoulos, and C. Papamanthou. vsql: Verifying arbitrary sql queries over dynamic outsourced databases. In Proceedings of the IEEE Symposium on Security and Privacy (S \& P), pages 863-880, 2017.

[82] Yupeng Zhang, Jonathan Katz, and Charalampos Papamanthou. Integridb: Verifiable sql for outsourced databases. In Proceedings of the Conference on Computer and Communications Security (CCS), pages 1480-1491, 2015.

[83] Qingji Zheng, Shouhuai $\mathrm{Xu}$, and Giuseppe Ateniese. Efficient query integrity for outsourced dynamic databases. In Proceedings of the ACM Workshop on Cloud computing security workshop, pages 71-82, 2012.

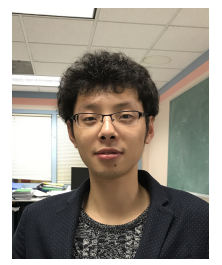

Bo Zhang was born in China in 1992. He received the B.S. from Wuhan University of Technology of China, Hubei, China. Currently he is a Ph.D. student of Stevens Institute of Technology, Hoboken, NJ, USA since 2015. His research interests include database, verifiable computation and data mining.

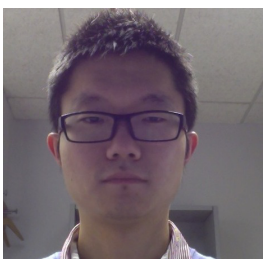

Boxiang Dong is an assistant professor in the Computer Science Department, Montclair State university, New Jersey. He received his Ph.D degree in computer science from Stevens Institute of Technology, New Jersey. He is dedicated to facilitating the integration of big data analysis and cybersecurity. His research interests include verifiable computing, data mining, anomaly detection, data security and privacy.

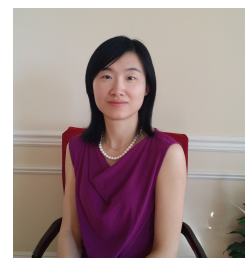

Dr. Wendy Hui Wang is an associate professor in the Computer Science Department, Stevens Institute of Technology, New Jersey. She received her $\mathrm{PhD}$ degree in computer science from University of British Columbia, Vancouver, Canada. Her research interests include data management, data mining, database security, and data privacy. She is a member of the editorial boards of Journal of Information Technology and Architecture and Journal of Information Systems. 